\title{
Participação das Mulheres na Graduação da Faculdade de Odontologia da Universidade Federal do Rio Grande do Sul
}

\author{
Women in School of Dentistry, Federal University of Rio Grande do Sul
}

\section{Renata Dos Santos Baldissera1, Fabiana Soares Grecca², Régis Burmeister dos Santos ${ }^{3}$}

\begin{abstract}
Introduction: In 1866, in the University of Ohio graduated the first woman in the history of Dentistry, The inclusion of women in dentistry has occurred slowly and gradually, following the historical and cultural development of society. One factor that makes women choose the dental career is being able to perform the work autonomously. Objective: Knowing the growth of women's participation in the Faculty of Dentistry, Federal University of Rio Grande do Sul (UFRGS-FO). Materials and Methods: The documents that contained all of the graduates of FO-UFRGS, in the period between 1900 and 2010, were analyzed. Results: In 1904, graduated the first three female students. In the early $60 \mathrm{~s}$, there was a steady increase in women's participation rate reached $45 \%$. In 2010, women constitute $68 \%$ of the students of the Faculty. Conclusions: We conclude that the woman started early on their participation in dentistry in the State. From this period until the present day there has been a gradual increase in this participation, culminating in their numerical predominance in relation to males.
\end{abstract}

Keywords: Dentistry; Women's work; Women.

\section{Resumo}

Introdução: Em 1866, na Universidade de Ohio, graduou-se a primeira mulher da história da Odontologia. A inserção da mulher na Odontologia ocorreu de forma lenta e gradual, acompanhando o desenvolvimento histórico e cultural da sociedade. Um dos fatores que faz com que a mulher opte pela carreira odontológica é o fato de poder exercer o trabalho de forma autônoma. Objetivo: Conhecer o crescimento da participação da mulher na Faculdade de Odontologia da Universidade Federal do Rio Grande do Sul (FO-UFRGS). Materiais e Métodos: Foram analisados os documentos que continham todos os formandos da FO-UFRGS do período de 1900-2010. Resultados: Em 1904, graduaram-se as três primeiras estudantes femininas. No inicio da década de 60, verificou-se um aumento crescente na participação da mulher atingindo taxa de $45 \%$. No ano de 2010 , as mulheres constituem $68 \%$ dos acadêmicos da Faculdade. Conclusões: Podemos concluir que a mulher iniciou cedo sua participação na Odontologia gaúcha. Deste período até os nossos dias tem havido um paulatino aumento desta participação, culminando com o seu predomínio numérico em relação ao sexo masculino.

Palavras chave: Odontologia; Trabalho feminino; Mulher.
1 Aluna de Gradução, Departamento de Odontologia Conservadora, Faculdade de Odontologia, Universidade Federal do Rio Grande do Sul

2 Doutora, Professora Adjunta, Departamento de Odontologia Conservadora, Faculdade de Odontologia, Universidade Federal do Rio Grande do Sul

3 Doutor, Professor Titular, Departamento de Odontologia Conservadora, Faculdade de Odontologia, Universidade Federal do Rio Grande do Sul

Correspondência: Fabiana Soares Grecca

Endereco: Rua Ramiro Barcelos, 2492 - 90035-003 - Porto Alegre - RS

Fone: +55 5133085191

E-mail: fabiana.grecca@ufrgs.br

Data de Submissão: 03/12/2010

Data de Aceite: 07/11/2011

\section{Introdução}

Este artigo tem como escopo trazer algumas reflexões sobre a participação feminina na carreira odontológica. Alguns dados gerais trazem informações sobre a mulher na odontologia dos Estados Unidos da América do Norte, nominando as pioneiras dos cursos de graduação e relacionando épocas desta ocorrência com o nosso país. O foco, contudo, é contribuir com algum entendimento da evolução da mulher no nosso meio, analisando a sua atuação nos bancos acadêmicos e a sua inclusão no magistério da Odontologia, avaliando quantitativamente seu crescimento na FO-UFRGS.

Desde os primórdios da atividade, por um longo período, os homens predominaram como profissionais da Odontologia. As mulheres participavam em atividades subalternas, auxiliando seus maridos ou, eventualmente, assumindo a profissão após o seu falecimento (RABELLO, 2000).

A inserção da mulher na Odontologia deu-se gradual e lentamente, acompanhando o desenvolvimento histórico e cultural da sociedade (RABELLO, 2000). A primeira faculdade de Odontologia foi criada em Baltimore (Maryland), em 1840. Em 1866, na Universidade de Ohio, graduou-se a primeira mulher da história da Odontologia (SILVA et al., 1996). No Brasil, após 15 anos da criação do curso de Odontologia na Faculdade de Medicina do Rio de Janeiro, em 1884, formou-se a primeira mulher, a paulista Isabella Von Sydow (GIFFIN, 1991).

A FO-UFRGS foi fundada em 10 de outubro de 1898 , tendo sido criada como um curso anexo à Faculdade de Medicina e Farmácia. Desde a fundação o seu estatuto, no artigo 39, já assegurava a matrícula de alunos do sexo feminino. As primeiras estudantes femininas a se graduarem foram: Alice Leão, Antônia Futuro e Nicolina Baltz no ano de 1904 (BRITO, 1998).

Em 1921 um grupo de 12 mulheres fundou a Associação Americana de Mulheres Dentistas com o objetivo de ter uma organização para partilhar interesses comuns, discutir e solucionar problemas profissionais (GIANGREGO, 1988; LOEVY et al., 1991).

Apesar da entrada da mulher no mercado de trabalho através de cursos superiores, o reconhecimento do valor social do trabalho feminino dava os primeiros passos e caminhava num ritmo mais lento, no século XIX. O trabalho feminino profissional, 'qualificado' e fora da esfera doméstica, não contava com tantos defensores, chamava a atenção e incomodava. O discurso dominante considerava o trabalho feminino válido desde que fosse 
realizado por mulheres solteiras e que tivesse como fim o suprimento das necessidades do lar. Deveria estar relacionado a profissões que exigissem qualidades consideradas inatas às mulheres (parteira, professora primária, enfermeira) ou voltadas para a clientela feminina e infantil (Medicina, Odontologia). Temiase 0 papel transformador do trabalho remunerado, a desorganização da família e a perda de espaço pelos homens no mercado de trabalho (MOTT, 2008).

Independentemente das objeções ao seu exercício profissional, as mulheres já estavam no mercado de trabalho. A entrada das mulheres em profissões tradicionalmente masculinas deveu-se a uma série de mudanças sociais, políticas, culturais e econômicas que vinham ocorrendo de forma cada vez mais rápida e acentuada a partir da segunda metade do século XIX (MOTT,2008).

A igualdade de oportunidades entre homens e mulheres tornou-se uma realidade em várias profissões (SPOSTO, 1997). A participação da mulher como força de trabalho, nas diferentes atividades humanas, tem, nos últimos tempos, sofrido considerável aumento percentual em relação aos homens, já relatava Silva et al. (1996). Este fato também é observado na área da saúde, especialmente na Odontologia. Essa realidade não é exclusiva do nosso país. Nos Estados Unidos um estudo feito por Taylor e Lovelace (1990) mostrou que o número de mulheres graduadas em Odontologia duplicou no período de 1979 a 1988, tendo aumentado de 634 para 1.263 cirurgiãs-dentistas. Para Rabello et al. (2000), uma razão para essa tendência é a situação econômica, que tem exigido a participação feminina para melhorar a condição financeira da família.

O aumento considerável do sexo feminino na área da saúde se deu após os anos setenta, o que foi constatado por Sposto et al. (1997) ao analisarem o número de homens e mulheres recém formados nos cursos de Odontologia de Faculdades públicas do Estado de São Paulo no período de 1950 1990.

Em 1980 houve uma troca na predominância do sexo masculino pelo feminino. Saliba et al. (2002) acreditam que a escolha da profissão se deve ao fato de as mulheres poderem conciliar a atividade profissional com as responsabilidades familiares. Aliadas as características da identidade feminina, a flexibilidade de horário, ausência de patrão e a compatibilidade com suas responsabilidades domésticas representam o "senso de conciliação" que induz a escolha por essa área, ao invés de outras mais complexas, como por exemplo, a engenharia, a química, a administração.

Neste sentido, também algumas pesquisas mostram que outro fator que induz a mulher a optar pela carreira odontológica é o fato de ela poder exercer o trabalho de forma autônoma. Esta circunstância permite às mulheres adequarem 0 exercício profissional ao seu estilo de vida, podendo compatibilizar o papel de mãe, esposa, dona de casa e profissional (COOMBS, 1976; DU, 1983; EPSON et al., 1974).

Segundo Saliba et al. (2002), a proporção de mulheres na Odontologia passou a ser majoritária dada às circunstâncias do contexto social e econômico do mundo contemporâneo. Apesar das diferenças culturais entre os países, a participação feminina no mercado de trabalho tem aumentado com a modernização da economia (ABURDENE, 1993).

Campos de trabalho que exploram a paciência, a observação, a minúcia e o "saber escutar" são constantemente justificados como sendo próprios das características da personalidade feminina. Por isso, os setores da educação, do lazer e da saúde, aqui representado pela Odontologia, são campos de maior atração para as mulheres conclui Saliba et al. (2002).

Apesar de atualmente ser uma profissão predominantemente de mulheres, algumas áreas ainda são consideradas masculinas como a Cirurgia Buco Maxilo Facial, enquanto outras como a Odontopediatria e a Dentística áreas femininas (PARAJARA, 2000).
Saliba et al. (2002), realizaram análise quantitativa sobre a participação do sexo feminino no período de 1961 a 2000 nas Faculdades de Odontologia de Araçatuba/Unesp e de Lins/Unimep. De 1961 a 1970 encontraram maior proporção masculina, onde $18,69 \%$ na Unesp e de $17,09 \%$ na Unimep, eram mulheres. No período de 1991 a 2000 o percentual ultrapassava a metade, sendo $59,95 \%$ de mulheres na Unesp e de $62,83 \%$ na Unimep. Os autores concluíram que vem ocorrendo um grande aumento do número de mulheres, o que aponta a preferência destas pela profissão, sendo necessário refletir sobre as conseqüências desse fato na prática odontológica.

Em uma análise dos registros dos graduados em Odontologia no Estado de Santa Catarina até os anos 80, Rosa e Almeida levantaram, em 1980, um panorama histórico no Estado. $\mathrm{Na}$ década de 20 a 30 formaram-se, em Santa Catarina, 14 mulheres, apesar de a Odontologia não ser considerada uma profissão de prestígio social para a época. Encontravam-se entre as profissões nobres a Medicina, a Engenharia e o Direito. Consideraram, além disso, a posição da mulher no contexto social, já que a profissão ideal para a mulher era a do lar. Estudar Odontologia não constituía uma atividade típica do sexo feminino. Já na década de 70 o ofício de cirurgião-dentista apresentou um crescimento, subindo aos primeiros escalões dentre as outras atividades profissionais. A situação das mulheres melhorou fazendo com que este período se tornasse, até então, o mais expressivo da sua participação na Odontologia catarinense.

Sob o título, Pesquisa Perfil Atual e Tendências do Dentista Brasileiro, o Ministério da Saúde e Observatório de RH em Odontologia da Faculdade de Odontologia da Universidade de São Paulo (FO-USP) divulgaram os primeiros resultados de uma pesquisa, onde, há 40 anos, as mulheres somavam $10 \%$ do total de cirurgiões-dentistas. Em 2009, constituem o percentual de $56 \%$ sendo maioria na profissão em 25 dos 27 estados brasileiros.

Em 1980, o Conselho Federal de Odontologia (CFO) publicou informações sobre o magistério na Odontologia, indicando que em 60 faculdades, Escolas ou Departamentos de Odontologia no Brasil existiam 4132 professores dos quais 755 (18,3\%) eram do sexo feminino.

Ao analisar a evolução da participação feminina na FOUFRGS, foram consultados documentos que continham todos os formandos no período de 1900-2010 e constatado que os números não diferem, de uma maneira geral, daqueles que foram até aqui expostos para outros lugares.

A partir de 1968, quando a FO passou a integrar a UFRGS, verificou-se um aumento crescente na participação da mulher, atingindo taxa de 45\%. No ano de 1976 dos 96 formandos, 66 foram mulheres (Gráfico 1).

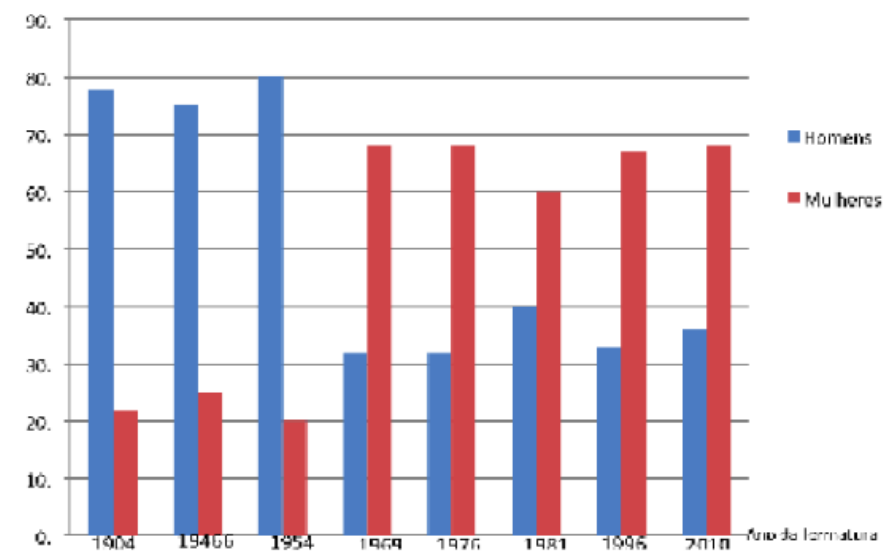

Gráfico 1- Distribuição da porcentagem de mulheres graduadas na FO-UFRGS em diferentes anos.

Contudo, diferente de outros estudos que apontam a participação da mulher como predominante nas duas últimas 
décadas (Saliba et al., 2002), na FO-UFRGS, em alguns anos como 2001 e 2003 verificamos que os homens ainda se apresentavam em maior número. Porém as taxas de formandas continuaram sendo altas (Gráfico 2).

Estão matriculados no ano 2010, 443 alunos, 303 do sexo feminino e 140 do sexo masculino. As mulheres constituem, portanto, $68 \%$ dos acadêmicos da Faculdade.

\section{Mulheres formadas}

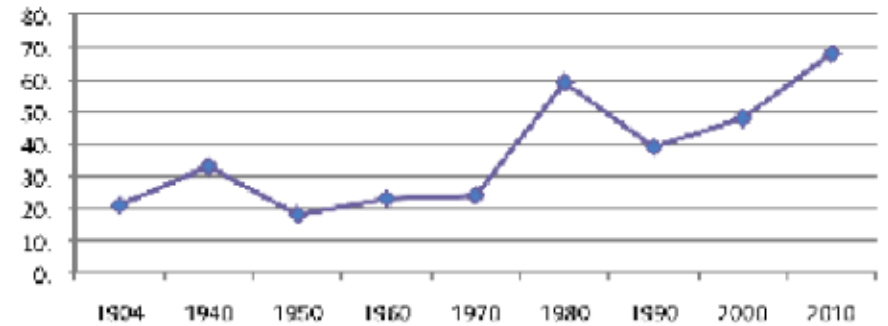

Gráfico 2- Percentagem de mulheres graduadas pela FO-UFRGS nas diferentes décadas.

Segundo Parajara (2000), o magistério foi outro reduto no qual o sexo feminino chegou aos poucos.

Trabalho realizado com professoras de Odontologia no Brasil, a fim de consultar sobre sua participação no contexto global do ensino superior, foi realizado por Silveira e Cordon em 1984. Através de um questionário, os autores levantaram informações sobre os aspectos mais importantes da situação da mulher no trabalho como docente da Odontologia. Foram respondidos 69 questionários, o que correspondeu a $50 \%$ das faculdades envolvidas na amostra. Quando perguntadas se tinham enfrentado problemas para tornarem-se professoras, $85 \%$ responderam negativamente e 13,2\% afirmativamente. As disciplinas mais procuradas pelas docentes foram a Odontopediatria e a Odontologia Social, seguidas de Ortodontia e Dentística.

$\mathrm{Na}$ FO-UFRGS, atualmente com um corpo docente de 81 professores, 43 são do sexo feminino. Assim distribuídas por departamento: Departamento de Odontologia Conservadora (DOC) com 20 professoras, sendo 04 da área de Dentística, com duas professoras as áreas de Endodontia, Materiais Dentários e Oclusão. Com 03 professoras a área de Prótese, Periodontia e Patologia, e com 01 professora as áreas de Estomatologia e Anatomia Dentária.

No Departamento de Odontologia Preventiva Social (DEOPS) estão 14 professoras. No Departamento de Cirurgia e Ortopedia (DCO) 09 professoras, sendo 03 das áreas de cirurgia, duas na área de Radiologia e com uma professora as disciplinas de Odontopediatria e Ortodontia (Gráfico 3).

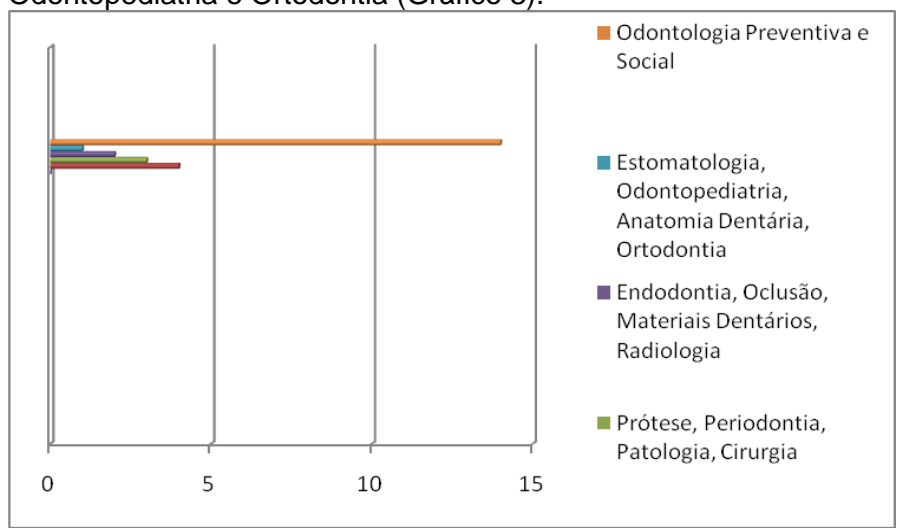

Gráfico 3- Quantidade de professoras por área.

A cultura gaúcha é propalada como machista e conservadora, entretanto, constata-se que as mulheres tiveram uma inserção precoce como acadêmicas de Odontologia no Estado. Fica claro, que, assim como nas outras atividades profissionais, a mulher entra efetivamente no mercado de trabalho a partir da década de 70. Deste período até os nossos dias tem havido um paulatino aumento desta participação, culminando com o seu predomínio numérico em relação ao sexo masculino.

\section{Referências}

ABURDENE, P. A revolução está aí. Veja, São Paulo, p. 7-10, 29 set. 1993

BRITO, J.H.M. Faculdade de Odontologia: cem anos de história 1898-1998. Porto Alegre: UFRGS, Fac. de Odontol., 1998.

CONSELHO FEDERAL DE ODONTOLOGIA. Pesquisa perfil atual e tendências do dentista brasileiro FO - USP 2009 Disponível em: < http://cfo.org.br/todas-as-noticias/noticias/previado-\%E2\%80\%9Cperfil-atual-e-tendencias-do-cirurgiao-dentistabrasileiro\%E2\%80\%9D/ >. Acesso em: 15 set. 2011.

CONSELHO FEDERAL DE ODONTOLOGIA. Relatório 1980. São Paulo: Gráfica Editora Hamburgo, 1980. 405 p

COOMBS, J.A. Factors associated with career choice among woman dental students. J. Dent. Educ., Washington, v. 40, no. 11, p. 724-732, Nov. 1976

DU, T. S. The woman dental student: a report. Diastema, Johannesburg, v. 11, p. 22-26, 1983.

EPSON, J.R.; DANIEL, N.; KELLER, E. Report on part-time work for women dentists with family commitments. Brit. Dental J., London, v. 136 , no. 12 , p. $513-514$, June 1974

GIANGREGO, E. AAWD: a voice for woman in dentistry. J. Am Dent. Assoc., Chicago, v. 117, no.3, p. 441-445, Sept. 1988

GIFFIN, K.M. Nosso corpo nos pertence: a dialética do biológico e do social. Cad. Saúde Pública, Rio de Janeiro, v. 7, n. 2, abr./jun. 1991.

LOEVY, H.T.; KOWITZ, A.A. Founders of the American Association of Women Dentists: their legacy ligers on. Int. Dent. J., London, $v$. 41, no. 4, p. 240-247, Aug. 1991.

MOTT, M. L. et al. Moças e senhoras dentistas: formação, titulação e mercado de trabalho nas primeiras décadas da República. Hist Cienc. Saúde-Manguinhos, Rio de Janeiro, v. 15, Supl., p. 97-116, 2008.

PARAJARA, F.A. Caminho da igualdade. Rev. Assoc. Paul. Cir. Dent., São Paulo, v. 54, n. 1, p. 11-19, jan./fev. 2000.

RABELLO, S.B. et al. Por que a odontologia se transformou numa profissão de mulher? Rev. Bras. Odontol., Rio de Janeiro, v. 57, n. 2, p. 118-123, mar./abr. 2000

ROSA, J.E.; MADEIRA, A.A. Participação da mulher na odontologia catarinense. Rev. Catarinense Odontol., Florianópolis, v. 7, n. 1, p. $19-25$, jan./jun. 1980 .

SALIBA, N.A. et al. Mulher na odontologia: uma análise quantitativa. Rev. Bras. Odontol., Rio de Janeiro, v. 59, n. 6, p. 400-402, nov./dez. 2002.

SILVA, E.M.; CRUZ, A.C.S. Por que as mulheres escolhem a odontologia? Rev. CROMG, Belo Horizonte, v. 2, n. 2, p. 65-69, jul./dez. 1996. 
SILVEIRA, H.; CORDON, J. A mulher no ensino superior em odontologia no Brasil. Rev. Fac. Odontol. Porto Alegre, Porto Alegre, v. 26, p. 25-36, 1984.

SPOSTO, M.R. et al. Evolução quantitativa da participação das mulheres nos cursos de odontologia das faculdades do eEstado de São Paulo (1950 - 1990). Rev. Bras. Odontol., Rio de Janeiro, v. 54, n. 2, p. 88-91, 1997.

TAYLOR, K.D.; LOVELACE, S.E. Slow passage to power. J. Calif. Dent. Assoc., Sacramento, v. 18, no. 6, p. 48-50, June 1990. 